\title{
Administration of Lactobacillus evokes coordinated changes in the intestinal expression profile of genes regulating energy homeostasis and immune phenotype in mice
}

\author{
Annika Nerstedt ${ }^{1}$, Elisabeth C. Nilsson ${ }^{1}$, Kajsa Ohlson ${ }^{2}$, Janet Håkansson ${ }^{2}$, L. Thomas Svensson ${ }^{1}$, \\ Björn Löwenadler ${ }^{1}$, Ulla K. Svensson ${ }^{2}$ and Margit Mahlapuu ${ }^{1 *}$ \\ ${ }^{1}$ Arexis AB, Göteborg, Sweden \\ ${ }^{2}$ Arla Foods, Innovation Center Stockholm, Stockholm, Sweden \\ (Received 16 June 2006 - Revised 23 October 2006 - Accepted 14 December 2006)
}

\begin{abstract}
Lactic acid bacteria are probiotics widely used in functional food products, with a variety of beneficial effects reported. Recently, intense research has been carried out to provide insight into the mechanism of the action of probiotic bacteria. We have used gene array technology to map the pattern of changes in the global gene expression profile of the host caused by Lactobacillus administration. Affymetrix microarrays were applied to comparatively characterize differences in gene transcription in the distal ileum of normal microflora (NMF) and germ-free (GF) mice evoked by oral administration of two Lactobacillus strains used in fermented dairy products today - Lactobacillus paracasei ssp. paracasei F19 (L. F19) or Lactobacillus acidophilus NCFB 1748. We show that feeding either of the two strains caused very similar effects on the transcriptional profile of the host. Both L. F19 and L. acidophilus NCFB 1748 evoked a complex response in the gut, reflected by differential regulation of a number of genes involved in essential physiological functions such as immune response, regulation of energy homeostasis and host defence. Notably, the changes in intestinal gene expression caused by Lactobacillus were different in the mice raised under GF $v$. NMF conditions, underlying the complex and dynamic nature of the host-commensal relationship. Differential expression of an array of genes described in this report evokes novel hypothesis of possible interactions between the probiotic bacteria and the host organism and warrants further studies to evaluate the functional significance of these transcriptional changes on the metabolic profile of the host.
\end{abstract}

Lactobacillus: Oligonucleotide microarray: Energy homeostasis: Immune regulation

During the last decades the role of the diet in development, as well as in prevention and management, of many diseases has been subjected to intense research. The term 'functional food' has been adapted to denote foods that may provide a health benefit beyond basic nutrition (Saris et al. 1998). The oldest and probably best-known functional food products are health-promoting bacteria or probiotics, defined as live microbial dietary supplements that beneficially affect consumers through their effects in the intestinal tract (Roberfroid, 2000). At present, probiotics, most often belonging to the genera Lactobacillus and Bifidobacteria, are almost exclusively consumed as fermented dairy products, such as yoghurt or freeze-dried cultures. Several health-related effects associated with the intake of probiotics have been reported in different animal models as well as in human studies (Roberfroid, 2000). However, the scientific evidence is still scarce and the mechanisms by which probiotics influence the host organism are only beginning to be explored.

The initial step in the characterization of mechanism of action for functional food products is the identification of a specific interaction between the active component of this food and an effect in the host organism that is potentially beneficial for health. One approach to investigate these interactions is to map the changes in transcription profile of the host organism caused by nutrient intake. Recent development of expression profiling by the use of microarray technology has made it possible to monitor the expression of thousands of genes simultaneously, allowing systematic analysis of complex biological processes and offering an advantage of reducing bias in data collection, compared with the candidate gene-based approaches. In the present report, we have studied the interactions between the intake of two strains of probiotic bacteria and regulation of intestinal gene expression of the host organism. Oligonucleotide microarrays were applied to compare global transcriptional profiles in the distal ileum of mice receiving Lactobacillus paracasei ssp. paracasei F19 (L. F19) or L. acidophilus NCFB 1748 with the control group of mice receiving a placebo product. The effects of the two Lactobacillus strains were evaluated both in germfree (GF) or normal microflora (NMF) mice. L. F19 as well as L. acidophilus NCFB 1748 has been used in products branded Cultura and Dofilus (Arla Foods, Stockholm,

Abbreviations: GF, germ-free; L. F19, Lactobacillus paracasei ssp. paracasei F19; NMF, normal microflora.

* Corresponding author: Margit Mahlapuu, fax +46 31749 1101, email margit.mahlapuu@ biovitrum.com 
Sweden). Both strains have good survival in the gastrointestinal tract, which is considered an important characteristic for the health-promoting activity of probiotics (Mättö et al. 2006). Previously, L. F19 and L. acidophilus NCFB 1748, in combination with Bifidobacterium lactis Bb12, have been shown to efficiently restore the intestinal microflora during antibiotic treatment (Sullivan et al. 2003). The objective of this study was to provide a comparative insight into the molecular mechanisms by which $L$. F19 and L. acidophilus NCFB 1748 interact with the host organism in a gnotobiotic environment $v$. in the context of the complex gut microflora.

\section{Materials and methods}

\section{Animal experiments}

GF and NMF male mice of Swiss Webster strain aged 6-8 weeks (Taconic, Lille Skensved, Denmark) were maintained under a standard 12-h light cycle regime, the relative humidity was between $45-55 \%$, the temperature was kept at $20^{\circ} \mathrm{C}$. The animals had ad libitum access to purified ingredient diet D12450B (Research Diets Inc., New Brunswick, NJ, USA) and water. NMF mice were housed in individually ventilated standard cages. GF mice were housed in gnotobiotic isolators and handled according to established procedures; isolators were daily monitored for sterility by culturing animal faeces and isolator interiors for bacteria. After 1 week of acclimatization, the mice were divided into two test groups, which received L. F19 ( $n$ 6) or L. acidophilus NCFB 1748 ( $n$ 6) bacteria in acidified ultra high-temperature processed-milk (approximately $5 \times 10^{8}$ colony forming units $/ \mathrm{ml}$ ) and a control group (four in NMF and five in GF), which received acidified milk only. In NMF conditions, the products were administrated twice per $\mathrm{d}$ during a 10-d period: the first dosing was performed by oral gavage ( $1 \mathrm{ml}$ per mouse) while the second dosing was done by sublingual injection $(100 \mu l$ per mouse). To minimize the risk of compromising the sterility of the gnotobiotic isolators, the number of product administration events was reduced in GF conditions: the products were fed to GF mice once per $d$ in 8 out of $10 \mathrm{~d}$ by oral gavage $(1 \mathrm{ml}$ per mouse). Following the period of product administration, the mice were killed by cervical dislocation. To avoid diurnal variations, all the mice were killed between 11.00 and 13.00 hours and approximately $4 \mathrm{~h}$ after the last dosing of Lactobacillus. The distal part of the ileum $(1.5 \mathrm{~cm})$ was excised for RNA extraction. Additional samples of ileum and colon were collected from each mouse for bacteriological analysis. To analyse the presence of $L$. F19 and $L$. acidophilus NCFB 1748 in the groups receiving an active product and the non-presence of these strains in the control groups, the samples were assayed on Rogosa agar (Merck, Darmstadt, Germany). Colonies were isolated and the presence of the two probiotic strains was confirmed by randomly amplified polymorphic DNA-PCR using the primers LBC-19 (5'-AGT AGC CAC-3') and OPA-05 (5'-TGC CGA GCT G-3') for screening and OPA-02 (5'-TGC CGA GCT G-3') and OPA-13 (5'-CAG CAC CAC-3') for confirmation of $L$. F19 and L. acidophilus NCFB 1748, respectively. Slanetz Bartley agar (Merck) was used for checking for the possible presence of enterococci. To confirm the absence of bacteria other than the administrated strains in ex-GF mice, these samples were spread on blood agar base (Oxoid, Basingstoke, Hants., UK) with defibrinated blood added to a final concentration of $7 \%$, and the plates were incubated both aerobically and anaerobically. The present study was performed after prior approval from the local ethical committee for animal experimentation.

\section{Test products}

Test products were produced from ultra high-temperature processed milk containing $1.5 \%$ fat. Probiotic organisms, L. F19 or L. acidophilus NCFB 1748, were added to a final concentration of $1 \times 10^{9}$ colony forming units $/ \mathrm{ml}$ and the milk was acidified to $\mathrm{pH} 4.5$ by addition of glucono- $\delta$-lactone to a final concentration of $1.6 \%$. The placebo product was identical to the active product despite no addition of probiotic organisms. The number of probiotic bacteria in the products was assayed by plating on MRS agar, pH 5.4 (de Man Rogosa Sharp; Oxoid). The absence of contaminating bacteria was confirmed by plating on count agar sugar free FIL-IDF (Fédération internationale de laiterie/International Dairy Federation) (Merck) and on plate count agar (Oxoid) with skimmed milk added to a final concentration of $0.1 \%$, for the active and placebo products, respectively.

\section{RNA extraction}

Tissues collected for RNA preparation were immediately submerged in RNAlater RNA Stabilization Reagent (Qiagen, Hilden, Germany) to preserve the quality and quantity of RNA. Total RNA was isolated from the intestinal tissue samples using the RNeasy Mini Kit (Qiagen) followed by a DNase digestion step (RNase-Free DNase Set; Qiagen) according to the manufacturer's instructions. The RNA yield was quantified by spectrophotometric analysis and the RNA purity was determined based on the $A_{260}: A_{280}$ ratio. All RNA samples were analysed by agarose gel electrophoresis to check for integrity of $18 \mathrm{~S}$ and $28 \mathrm{~S}$ rRNA. Further, the quality of the RNA was verified by Agilent 2100 Bioanalyzer analysis (Agilent Technologies, Palo Alto, CA, USA) using the RNA 6000 Nano Assay Kit (Agilent Technologies).

\section{Expression profiling by Affymetrix}

Total RNA spiked with poly-A controls (pGIBS-TRP, - THR and -LYS; American Type Culture Collection, Manassas, VA, USA) was converted into cDNA utilizing a T7 promoter-poly T primer (Genset, Paris, France) and the RT Superscript II (Invitrogen Life Technologies, Paisley, UK) followed by a second strand cDNA synthesis (Invitrogen Life Technologies). ds cDNA was in vitro transcribed into biotinylated cRNA (Enzo Life Sciences, Farmingdale, NY, USA). Finally, fragmented cRNA (35-200 bases) was used as a target for hybridization. Hybridization spike controls (oligonucleotide B2 and a cRNA cocktail (BioB, BioC, BioD and Cre; GeneChip Eukaryotic Hybridization Control Kit; Affymetrix, Santa Clara, CA, USA)) were used as hybridization quality controls. Aliquots of each sample were hybridized $\left(16 \mathrm{~h}\right.$ at $45^{\circ} \mathrm{C}$ ) to the Murine Genome Array U74Av2 (Affymetrix). The arrays were subsequently washed, stained and scanned according to the manufacturer's instructions (GeneChip Expression Analysis Technical Manual; Affymetrix). Data were analysed using robust multi-chip analysis in GeneTraffic UNO version 2.6-25 (Stratagene, La Jolla, CA, USA) and Spotfire 
DecitionSite for Functional Genomics version 7.1 (Spotfire Inc., Göteborg, Sweden). The $\log _{2}$ fold change for each sample and probe set $v$. the control group was calculated using the formula: $\log _{2}$ fold change $=\log _{2}$ (probe set intensity/mean probe set intensity in the control group). The mean $\log _{2}$ fold change was calculated for each Lactobacillus strain $v$. the control group. Statistical significance of the difference in gene expression was determined using two-sided Student's $t$ test. A transcript was considered differentially expressed if the mean absolute $\log _{2}$ fold change was $>0.5$ (corresponding to a mean absolute fold change $>1.41)$ and the $P$-value was $<0 \cdot 05$. In addition, the mean intensity in the group showing the highest expression should be $>75$. To view the microarray data in a biological context, Ingenuity Pathway Analysis Software tool (Ingenuity Systems, Redwood City, CA, USA; http: Iwwww.ingenuity.com) was used to generate a network connecting the differentially regulated targets and all other gene products, based on known mammalian gene product events (such as protein-protein, proteinnucleic acid interactions) defined in Ingenuity System's database.

Expression profiles of genes differentially regulated in GF mice in response to Lactobacillus administration were compared with the corresponding profiles in GF mice of the NMRI/KI strain mono-colonized by Bacteroides thetaiotaomicron, using the data published by Hooper et al. (2001). The .CEL files from this study (Mu11K and Mu19K Affymetrix array sets, duplicate microarray hybridizations performed on pooled ileal RNA samples corresponding to GF $v$. mono-colonized mice) kindly provided by Professor L.V. Hooper, were re-analysed using Microarray Suite Version 5.0 (MAS5.0) (Affymetrix) and Spotfire DecitionSite for Functional Genomics version 7.1 (Spotfire Inc.). The overall intensity across each array was scaled to a target intensity of 150. A transcript was considered differentially expressed if the mean absolute $\log _{2}$ fold change was $>0.5$ (corresponding to a mean absolute fold change $>1.41$ ). Additionally, the mRNA should be called Present by the MAS5.0 software in either GF or colonized mice, and the differential expression should be observed in all four comparisons performed on duplicate microarray hybridizations (see Hooper et al. (2001) for details concerning the design of the microarray experiment).

\section{Quantitative real-time PCR}

Expression profiles for selected targets were confirmed using quantitative real-time PCR with the ABI PRISM 7000 Sequence Detector System (Applied Biosystems, Foster City,
CA, USA), according to the manufacturer's instructions. Briefly, total RNA, isolated for the expression profiling, was converted into cDNA by utilizing the Superscript ${ }^{\mathrm{TM}}$ firststrand synthesis system (Invitrogen Life Technologies). PCR reactions $(25 \mu \mathrm{l})$ contained each PCR primer $(400 \mathrm{~nm})$ designed by using PRIMER EXPRESS 2.0 software (Applied Biosystems) and 1x SYBR ${ }^{\circledR}$ Green PCR Master Mix (Applied Biosystems). The forward and reverse primer sequences are presented in Table 1. All reactions were performed in duplicate and a dissociation curve was completed for every PCR run to control the specificity of the amplification reaction. The relative quantities of different mRNA transcripts were calculated after normalization of the data against an endogenous control - acidic ribosomal phosphoprotein P0 (Arbp) using the standard curve method (Applied Biosystems, 1997).

\section{Results}

Microarray analysis of the differential gene expression evoked by oral administration of Lactobacillus strains

We have used oligonucleotide microarray to analyse the host transcriptional responses caused by oral delivery of Lactobacillus bacteria. The test groups of mice received one strain of Lactobacillus - L. F19 or L. acidophilus NCFB 1748 - in acidified milk, while the control group of mice received acidified milk only. Experiments were performed using age-matched male mice raised under GF or NMF conditions. After the period of product administration of $10 \mathrm{~d}$, the presence of the corresponding Lactobacillus strains in the intestinal contents of the test groups of mice, and the lack of these bacteria in the control group, were confirmed. Importantly, from the intestine of the test groups of mice raised under GF conditions, only the administrated strain could be re-isolated, while the corresponding control group remained GF during the whole study period. While previous studies have shown that the different strains of Lactobacillus bacteria are able to colonize the whole gastrointestinal tract of GF mice and are established in high numbers in both the small and large intestine (Wagner et al. 1997; Ibnou-Zekri et al. 2003), we found that L. F19 and L. acidophilus NCFB 1748 were present in the ileum of the GF mice in considerably larger numbers than compared with the colon (data not shown). The gene expression profiles in the distal ileum of the test groups of mice were compared with the corresponding control group using Affymetrix gene arrays.

Table 1. Primer sequences used for quantitative real-time-PCR analysis

\begin{tabular}{llll}
\hline Gene & \multicolumn{1}{c}{ Forward primer $\left(5^{\prime}-3^{\prime}\right)$} & \multicolumn{1}{c}{ Reverse primer $\left(5^{\prime}-3^{\prime}\right)$} & Accession no. \\
\hline Clu & CCACCGTGACCACCCATT & CAGCTTCACCACCACCTCAGT & NM_013492 \\
$l g h-6$ & ACTGCCTCCACCTTCATCGT & CTGAGAGTCATTTCACCTTGAACAG & BC098504 \\
Cxcl13 & AACGCAGGCTTCCAAAATAGTC & TGCTTTGCACCACCTCATGA & NM_018866 \\
Ltb & ATCGGGTACGGGTCGTTATG & ATCACCGCCCCGAAGAAG & NM_008518 \\
Serpina1c & CAAACTCTCAGCAAGGAGCTCAT & GGGAAGTGGATCTGGGCTAAC & NM_009245 \\
Rbp2 & ACATGAAGGCCCTAGATATTGATTTT & AGTGATGATCTTCGTCTGAGTCAGA & NM_009034 \\
Apoa4 & CAGCTGGGTCCCAATTCG & CAGGGTGCTCATAAAGGAGTTGA & NM_007468 \\
Retnlb & CAATGCTCCTTTGAGTCTTTGGT & GCAGGAGATCGTCTTAGGCTCTT & NM_023881 \\
Adipoq & TCAACGACTCTACATTTACTGGCTTT & GTTCCATGATTCTCCTGGTGTATG & NM_009605 \\
Cfd & GCTATCCCAGAATGCCTCGTT & GGTTCCACTTCTTTGTCCTCGTA & NM_013459 \\
Car3 & CACACGTTAACATCATTGTAGATCTCA & CTTGGTAGTAGGCAAATTTTAACGA & NM_007606 \\
Arbp & GAGGAATCAGATGAGGATATGGGA & AAGCAGGCTGACTTGGTTGC & NM_007475 \\
\hline
\end{tabular}


The global gene expression analysis demonstrated that genes altered by administration of Lactobacillus are involved in widely different functions. In the NMF mice, the expression of twenty-two probe sets was significantly $(P<0 \cdot 05)$ changed by a factor of more than $41 \%$ (absolute $\log _{2}$ fold change $>0.5)$, in at least one test group, relative to the control group (Table 2). Administration of Lactobacillus to GF mice led to the differential expression of thirty probe sets in one or both test groups, relative to control mice (absolute fold change $>1.41, P<0.05$; Table 3 ). From these transcripts, the identity of most of the genes is known and represents proteins of different functional classes, whereas three transcripts only show homology to sequences in the Expressed sequence tag (EST) or genomic databases. The vast majority of genes, which were significantly changed exclusively in mice receiving L. F19 or L. acidophilus NCFB 1748, tended to be regulated in the same manner in the other test group, even though this difference did not reach statistical significance and/or meet the fold change criteria. Importantly, transcriptional changes evoked by administration of Lactobacillus to NMF mice were different from the responses seen in GF mice. In fact, only one probe set, representing retinol binding protein 2, showed significant difference in response to the delivery of Lactobacillus bacteria both to NMF and GF mice (Tables 2 and 3).

\section{Quantitative real-time PCR validation of differentially expressed genes}

To minimize erroneous conclusions due to technical variability and multiple testing effects inherent to the microarray technology, quantitative real-time PCR analysis was applied to validate expression profiles of eleven genes selected on the basis of biological interest (Fig. 1). For all the genes examined, quantitative real-time PCR data are in good agreement with the gene array results with regard to the direction of observed changes. Furthermore, individual animal-to-animal comparison of the expression profiles for these genes showed good correlation comparing the two techniques (data not shown).

\section{Administration of Lactobacillus to normal microflora mice modulates the immune phenotype expression}

Several genes with a potential role in the intestinal immune system - Clu, C3, Bcl6, Ptprc, Serpinal, Laptm5 and Vcam1 - were significantly up-regulated in the NMF mice receiving L. acidophilus NCFB 1748 and showed identical transcription profiles. Searching for similar expression patterns using Profile Search (Spotfire DecitionSite for Functional Genomics version 7.1; Spotfire Inc.) with correlation as similarity measure ( 0.92 as cut off) identified thirty-five additional probe sets up-regulated by a factor of more than $41 \%$ in at least one test group, compared with the control mice (Table 2). Ingenuity Pathway Analysis (Ingenuity Systems) reveals that these targets form an integrated functional network controlling different aspects of immune system development and function and are regulated by common upstream factors (Fig. 2(A)). Most of the transcripts in this cluster are expressed predominantly or exclusively in B cells, e.g. the components of the B cell receptor for antigen including Ig molecule (encoded by Ighs), $C d 79 a$ and $C d 79 b$, as well as factors involved in downstream signalling from the B cell receptor, such as Ptprc (alias Cd45), Cd19, Blk, Lck and Prkcb1. A key mediator responsible for the organization of B cells within lymphoid structures, $L t b$, together with three additional markers potentially involved in this function, Cxcl13, Vcaml and $B c l 6$, were up-regulated in response to Lactobacillus administration. Additionally, genes with potential function in phagocytosis (Mfge8 and Corola) and complement function (C3 and $\mathrm{Clu}$ ) were increased in the test groups. Several probe sets supported an up-regulation in Serpinal transcript, encoding for $\alpha 1$-antitrypsin, in response to Lactobacillus administration.

\section{Administration of Lactobacillus strains in germ-free mice evokes coordinated changes in expression profiles of genes regulating energy homeostasis and host defence}

Three transcripts encoding for key regulators of fat and sugar metabolism and insulin sensitivity at the whole body level adipsin $(C f d)$, adiponection (Adipoq) and resistin like $\beta$ $($ Retnlb) - were differentially expressed in the test groups of mice compared with the control group (see Table 3 for statistical significance notes). Adipsin and adiponectin, an insulin-sensitizing hormone (Pajvani \& Scherer, 2003), were up-regulated, while resistin like $\beta$, known to induce insulin resistance (Rajala et al. 2003), was down-regulated, in response to Lactobacillus administration. Transcripts for three cytosolic proteins, Scd1, Car3 and Thrsp, with possible functions in lipid metabolism, were significantly up-regulated in the groups of mice receiving Lactobacillus (Table 3). Stearoyl-Coenzyme A desaturase 1 is an Fe-containing enzyme that catalyses a rate-limiting step in the synthesis of unsaturated fatty acids. Although very little is known about the specific function of Car3 and Thrsp, both genes are implicated in fatty acid metabolism (Stanton et al. 1991; Kinlaw et al. 1995). Additionally, several genes with a potential role in intestinal defence against bacterial infections - Mmp7, Lyzs, Pla2g2a, Sprrla, Igh6 - were up-regulated in response to Lactobacillus administration in GF mice. Ingenuity Pathway Analysis (Ingenuity Systems) indicated that the differentially expressed targets involved in regulation of energy homeostasis as well as host defence form a complex network regulated by common upstream mediators, with TNF having a central regulatory role (Fig. 2(B)).

To evaluate if the changes in gene expression profile we describe are specific to Lactobacillus or can be elicited by inoculation of the gnotobiotic intestine with non-probiotic bacteria as well, we turned to the previously published study describing the effects of colonization with Bacteroides thetaiotaomicron, a prominent component of the normal intestinal microflora (Hooper et al. 2001). In this report, the global gene expression profile in the distal ileum of mice inoculated with Bacteroides was compared with the age-matched mice remaining GF by Affymetrix microarrays, thus providing a good comparison to our dataset. We compared the transcription profiles of genes differentially regulated in response to Lactobacillus (Table 3) with the corresponding profiles in mice colonized by Bacteroides, using the raw data files kindly provided by Professor L.V. Hooper (see Materials and Methods for details on data analysis). The probe sets for 
Table 2. Differentially expressed genes in normal microflora mice receiving Lactobacillus paracasei ssp. paracasei $\mathrm{F} 19$ (F19) or Lactobacillus acidophilus NCFB 1748 (NCFB) compared with the control group of mice receiving placebo product $\$$

\begin{tabular}{|c|c|c|c|c|}
\hline \multirow[b]{2}{*}{ Probe set ID } & \multirow[b]{2}{*}{ Gene symbol } & \multicolumn{2}{|c|}{ Fold change } & \multirow[b]{2}{*}{ Potential function } \\
\hline & & F19 & NCFB & \\
\hline \multicolumn{5}{|c|}{ Immune regulation } \\
\hline 92740_at† & $\operatorname{lgh}-5$ & $2 \cdot 13$ & $2 \cdot 61$ & Ig heavy chain of IgD \\
\hline 93584_at† & Igh-6, MGC60843 & 1.67 & 2.41 & Ig heavy chain of IgM \\
\hline 99446_at† & Ms4a1 & $1 \cdot 71$ & $2 \cdot 27$ & $\begin{array}{l}\text { a B-lymphocyte surface marker involved in regulation of B-cell } \\
\text { proliferation and differentiation }\end{array}$ \\
\hline 95286_at & $\mathrm{Clu}$ & 1.75 & $2 \cdot 24^{\star}$ & a multifunctional glycoprotein involved in complement regulation \\
\hline 102025_at & Cxcl13 & 1.85 & $2 \cdot 23$ & $\begin{array}{l}\text { a chemokine required for the architectural organization of B-cells } \\
\text { within lymphoid follicles }\end{array}$ \\
\hline 102940_at† & $L t b$ & 1.50 & 1.95 & $\begin{array}{l}\text { a membrane protein involved in organization of secondary } \\
\text { lymphoid structures in the intestine }\end{array}$ \\
\hline 93583_s_at† & Igh-6, MGC60843 & 1.57 & 1.90 & Ig heavy chain of IgM \\
\hline 100468_g_at & Lyl1 & 1.44 & 1.83 & a cytosolic protein expressed in most B-lineage cells \\
\hline 101048_at & Ptprc & 1.49 & $1 \cdot 81^{*}$ & $\begin{array}{l}\text { a transmembrane tyrosine phosphatase, positive regulator } \\
\text { of BCR signalling }\end{array}$ \\
\hline 92880_at + & Mfge8 & 1.59 & 1.74 & a secreted glycoprotein involved in phagocytosis \\
\hline 161294_f_at† & Clu & 1.56 & $1 \cdot 70$ & a multifunctional glycoprotein involved in complement regulation \\
\hline 102778_at $†$ & Cd79a & $1 \cdot 68$ & 1.67 & $\begin{array}{l}\text { a B-lineage-specific member of the Ig superfamily, together with } \\
\text { CD79b forms the signal transducing part of the BCR }\end{array}$ \\
\hline 95893_at† & $B / k$ & 1.45 & 1.65 & a Src-family protein tyrosine kinase activated by BCR \\
\hline 94278_at $\dagger$ & Lcp1 & 1.48 & 1.63 & a major lymphocyte cytosolic protein \\
\hline 93497_at & C3 & 1.51 & $1 \cdot 62^{*}$ & $\begin{array}{l}\text { complement factor, complement-coated antigens cross-link CD21 } \\
\text { with the BCR increasing the signal through the receptor }\end{array}$ \\
\hline 96648_at & Coro1a & 1.43 & 1.59 & an actin-binding protein required for phagosome formation \\
\hline 100329_at & Serpina1c & 1.58 & $1.51^{*}$ & a protease inhibitor, implicated in protection against mucosal \\
\hline 101576_f_at† & Serpina $1 b$ & 1.53 & 1.55 & damage in inflammatory bowel disease \\
\hline 93109_f_at† & Serpina1a,b,c,d & 1.47 & 1.53 & \\
\hline 161012_at & $C d 79 b$ & $1 \cdot 40$ & 1.83 & $\begin{array}{l}\text { a B-lineage-specific member of the Ig superfamily, together with } \\
\text { CD79a forms the signal transducing part of the BCR }\end{array}$ \\
\hline 93915_at† & Pou2af1 & $1 \cdot 29$ & 1.77 & $\begin{array}{l}\text { B-cell-specific transcriptional co-activator regulating expression of } \\
\text { lg genes }\end{array}$ \\
\hline 104606_at† & Cd52 & 1.35 & $1 \cdot 74$ & $\begin{array}{l}\text { a cell surface protein expressed in lymphocytes, macrophages } \\
\text { and monocytes }\end{array}$ \\
\hline 102823_at† & lghg & 1.23 & 1.58 & Ig heavy chain of IgG \\
\hline 103015_at & Bcl6 & 1.39 & $1 \cdot 56^{*}$ & $\begin{array}{l}\text { a transcriptional repressor expressed in B-cells, controls germinal } \\
\text { centre formation }\end{array}$ \\
\hline 94 939_at† & Cd53 & 1.37 & 1.56 & a glycoprotein expressed in leukocytes \\
\hline 100012_at & Laptm5 & 1.41 & $1.55^{\star}$ & a lysosomal protein expressed mostly in haematopoietic cells \\
\hline 102824_g_at† & lghg & 1.23 & 1.54 & Ig heavy chain of IgG \\
\hline 102809_s_at† & Lck & 1.37 & 1.52 & a Src-family protein tyrosine kinase activated by BCR signalling \\
\hline 92558_at & Vcam1 & 1.33 & $1.52^{*}$ & $\begin{array}{l}\text { a cell surface glycoprotein expressed in endothelium, where it } \\
\text { mediates the adhesion of monocytes and lymphocytes }\end{array}$ \\
\hline 103040_at $†$ & Cd83 & 1.35 & 1.51 & a cell surface molecule expressed at haematopoietic cells \\
\hline 93957_at & Vpreb3 & 1.35 & 1.50 & $\begin{array}{l}\text { expressed exclusively in B-cells, associates with membrane Ig } \\
\text { heavy chains early in the course of BCR biosynthesis }\end{array}$ \\
\hline 98980_at† & Cd37 & $1 \cdot 34$ & 1.47 & $\begin{array}{l}\text { a membrane protein expressed predominantly on the surface } \\
\text { of } B \text { cells }\end{array}$ \\
\hline 101876_s_at & H2-T10,H2-T22,H2-T9 & $1 \cdot 23^{\star *}$ & $1.47^{*}$ & belongs to the major histocompatibility complex, class II \\
\hline 92741_g_at† & $\operatorname{lgh}-5$ & 1.46 & 1.36 & Ig heavy chain of IgD \\
\hline 97994_at† & Tcf7 & 1.35 & 1.46 & $\begin{array}{l}\text { a T-cell-specific transcription factor, which controls thymocyte } \\
\text { differentiation }\end{array}$ \\
\hline 99945_at† & Cd19 & 1.40 & 1.45 & $\begin{array}{l}\text { a transmembrane protein associated with BCR, acts as a adaptor } \\
\text { molecule and amplifies BCR signals }\end{array}$ \\
\hline 103231_at† & Rhoh & $1 \cdot 31$ & 1.45 & $\begin{array}{l}\text { a protein similar to members of the Ras superfamily, expressed } \\
\text { in haemopoietic cell lines only }\end{array}$ \\
\hline 99510_at† & Prkcb1 & 1.39 & 1.44 & $\begin{array}{l}\text { a kinase functionally linked to Bruton kinase in BCR-mediated } \\
\text { signal transduction }\end{array}$ \\
\hline 102851_s_at† & Ptpn6 & $1 \cdot 30$ & 1.43 & $\begin{array}{l}\text { a tyrosine protein phsophatase expressed predominantly } \\
\text { in haematopoietic cells }\end{array}$ \\
\hline 96172 at† & Gimap4 & 1.37 & 1.42 & a protein expressed in B and T cells, function not clear \\
\hline 100377_f_at & $\operatorname{lghmAC} 38.205 .12$ & -1.01 & $-1 \cdot 69^{*}$ & Ig heavy chain of IgM \\
\hline 93638_s_at & $|g|-V 1$ & $-1 \cdot 67^{*}$ & $-1 \cdot 24$ & $\lg \lambda$ chain, variable region \\
\hline 100360 f_at & $\operatorname{lgh}-4$ & $-1 \cdot 15$ & $-1 \cdot 64^{*}$ & Ig heavy chain for serum IgG1 \\
\hline $97009 \_$f_at & lgh-V & 1.00 & $-1 \cdot 61^{*}$ & Ig heavy chain, variable region \\
\hline \multicolumn{5}{|l|}{ Miscellaneoous } \\
\hline 100078_at & Apoa4 & $1 \cdot 18$ & & $\begin{array}{l}\text { a satiety signal secreted by the small intestine, implicated in } \\
\text { regulation of both short and long-term food intake }\end{array}$ \\
\hline 95673_s_at† & Basp1 & $1 \cdot 20$ & $1 \cdot 68$ & expressed in nervous tissue, function largely unknown \\
\hline
\end{tabular}


Table 2. Continued

\begin{tabular}{|c|c|c|c|c|}
\hline \multirow[b]{2}{*}{ Probe set ID } & \multirow[b]{2}{*}{ Gene symbol } & \multicolumn{2}{|c|}{ Fold change } & \multirow[b]{2}{*}{ Potential function } \\
\hline & & F19 & NCFB & \\
\hline 94540_at & Cyp2d26 & $1 \cdot 20$ & 1.58 & $\begin{array}{l}\text { a member of cytochrome P450 family, specific function poorly } \\
\text { understood }\end{array}$ \\
\hline 94004_at & Cnn2 & 1.31 & $1.57^{*}$ & widely expressed, function largely unknown \\
\hline 101972_at† & Napsa & 1.37 & 1.49 & an aspartic proteinase, function not clear \\
\hline 93874_s_at & II11ra1, I/11ra2 & $1 \cdot 12$ & $1.48^{* *}$ & $\begin{array}{l}\text { a receptor for IL-11, which is a stromal cell-derived cytokine } \\
\text { with multiple biological activities }\end{array}$ \\
\hline 104707_at & Tm4sf5 & 1.06 & $1.46^{*}$ & a cell surface protein, function poorly described \\
\hline 92811_at & Rbp2 & $1 \cdot 21$ & $1.43^{*}$ & regulates the uptake and metabolism of vitamin $\mathrm{A}$ \\
\hline 160308_at $†$ & Msn & $1 \cdot 35$ & 1.45 & $\begin{array}{l}\text { expressed in different tissues, localizes to membrane protrusions } \\
\text { that are important to cell-cell recognition, signalling and } \\
\text { cell movement }\end{array}$ \\
\hline 96353_at & Tmem14c & $-2 \cdot 54^{*}$ & -1.54 & transmembrane protein, function poorly described \\
\hline 93934_at & Cldn2 & -1.05 & $-1.52^{*}$ & $\begin{array}{l}\text { a member of a claudin family of integral membrane proteins } \\
\text { localized at tight junctions }\end{array}$ \\
\hline 100946_at & Hspa1b & -1.07 & $-1 \cdot 48^{*}$ & $\begin{array}{l}\text { a heat-shock protein, expressed in response to heat shock and a } \\
\text { variety of other stress stimuli }\end{array}$ \\
\hline 98384_at & Ptk6 & -1.09 & $-1 \cdot 43^{*}$ & a kinase implicated in cell transformation \\
\hline
\end{tabular}

Values were significantly different, determined by two-sided Student's $t$ test: $\left({ }^{\star} P \leq 0.05 ;{ }^{* *} P \leq 0.01\right)$.

$\dagger$ Transcripts were identified by similarity search using mean expression profile of Clu, C3, Bcl6, Ptprc, Serpina1, Laptm5 and Vcam1 as a template.

$\ddagger$ Global mRNA expression pattern was characterized in distal ileum using Affymetrix (Santa Clara, CA, USA) gene arrays. The filtering criteria were set to a mean absolute fold change $>1.41\left(\log _{2}\right.$ fold change $\left.>0.5\right)$ and a $P$ value $<0.05$ in either of the two test groups, compared with the control group. In addition, the mean intensity in the group showing highest expression should be $>75$.

$\S$ For details of procedures, see Materials and methods.

The colours represent the differential expression pattern. Green indicates down-regulation; red indicates up-regulation with an absolute log fold $_{2}$ change $>0.5$. BCR, B cell receptor.

several genes involved in regulation of energy homeostasis (Adipoq, Retnlb, Scd1) were represented and called Present on these arrays; however, none of these transcripts was regulated in response to Bacterioides in the manner similar to Lactobacillus. On the contrary, two probe sets supported an up-regulation of Retnlb by Bacteroides, while this gene was down-regulated in response to Lactobacillus. Interestingly, $R b p 2$, encoding a protein shown to participate in uptake and metabolism of vitamin A in the small intestine (Levin, 1993; Lissoos et al. 1995), was up-regulated in response to Bacteroides as well as Lactobacillus. Similar to Lactobacillus, administration of Bacteroides increased expression of genes implicated in host defence, including small proline like proteins (Sprr2a up-regulated by Bacteroides and Sprrla by Lactobacillus) and Pla2g2a (data not shown).

\section{Discussion}

In the present study, we investigated the molecular effects of the two Lactobacillus strains - L. F19 and L. acidophilus NCFB 1748 - on global gene expression profile in the distal small intestine using the Affymetrix gene arrays. The changes in transcriptional profile caused by oral administration of Lactobacillus bacteria in NMF mice were compared with the effects evoked by administration of these strains in GF mice, with the GF mice representing a simplified model of interactions between gut commensals and their host.

Lactobacilli have long been acknowledged to promote the intestine's immunological barrier, particularly through enhancement of humoral immune responses, induction of germinal centre formation, activation of the phagocytosis by macrophages and alleviation of intestinal inflammatory responses (Wagner et al. 1997; Perdigon et al. 1999; Shu \& Gill, 2002; Ibnou-Zekri et al. 2003; Galdeano \& Perdigon,
2006). Recent data indicate that differences may exist in the immune stimulatory effects of specific strains of probiotic bacteria (Perdigon et al. 1999; Ibnou-Zekri et al. 2003). In line with the previous reports, the present study demonstrated that the administration of two Lactobacillus strains to NMF mice caused concerted increases in a cluster of genes involved in immune response (Table 2, Fig. 2(A)). Several components of B cell receptor-signalling were up-regulated (Ighs, Cd79a, Cd79b, Ptprc (alias Cd45), Cd19, Blk, Lck, Prkcb1), suggesting mobilization of B-lymphocytes. Also, transcripts implicated in phagocytosis (Mfge8, Corola), complement function $(\mathrm{C3}, \mathrm{Clu})$ and architectural organization of $B$ cells within lymphoid structures ( $L t b, C x c l 13, V c a m 1$ and Bcl6) were increased in response to Lactobacillus administration. Previously, Di Caro et al. (2005) demonstrated by global gene expression profiling in duodenal mucosa that administration of L. rhamnosus to human subjects induced expression of a number of genes involved in immune response, including $\mathrm{Ltb}, \mathrm{Cxcl13}, \mathrm{C} 3$ and Ms4al, which were also up-regulated by the two strains used in the present study. We did not detect any qualitative differences comparing the effect of L. F19 v. L. acidophilus NCFB 1748 on the expression profile of immune response-related genes. However, the mean fold change of the increased signal for this group of transcripts was higher in mice receiving L. acidophilus NCFB 1748. Notably, immune stimulatory effect in response to Lactobacillus administration was not observed in mice raised under GF conditions. The intestinal microflora has a large impact on the development of gut-associated lymphoid tissue. Also, the response to probiotic bacteria depends on the immunological state of the host (Falk et al. 1998). Therefore, we speculate that under the conditions used, the intestinal tissue of gnotobiotic mice might have been incompetent to respond to the stimulation by lactic acid bacteria as observed in NMF mice. 
Table 3. Differentially expressed genes in germ-free mice receiving Lactobacillus paracasei ssp. paracasei F19 (F19) or Lactobacillus acidophilus NCFB 1748 (NCFB) compared with the control group of mice receiving placebo product†‡

\begin{tabular}{|c|c|c|c|c|}
\hline \multirow[b]{2}{*}{ Probe set ID } & \multirow[b]{2}{*}{ Gene symbol } & \multicolumn{2}{|c|}{ Fold change } & \multirow[b]{2}{*}{ Potential function } \\
\hline & & F19 & NCFB & \\
\hline \multicolumn{5}{|c|}{ Energy homeostasis } \\
\hline 99671_at & Cfd & 1.59 & 2.35 & regulates insulin sensitivity \\
\hline 99 104_at & Adipoq & 1.76 & $2 \cdot 26^{*}$ & regulates insulin sensitivity \\
\hline 160375_at & Car3 & 1.70 & $2 \cdot 17^{*}$ & implicated in fatty acid metabolism \\
\hline 94057 g_at & Scd1 & 1.78 & $1.95^{*}$ & catalyses a rate-limiting step in the synthesis of unsaturated \\
\hline 94 056_at & Scd1 & 1.63 & $1 \cdot 80^{*}$ & fatty acids \\
\hline 160306_at & Thrsp & $1.55^{*}$ & $1.25^{* *}$ & implicated in lipogenesis \\
\hline 93755_at & Retnlb & $-29 \cdot 33^{*}$ & $-25 \cdot 14^{*}$ & antagonizes insulin action \\
\hline \multicolumn{5}{|l|}{ Host defence } \\
\hline 92917_at & Mmp7 & $2 \cdot 26$ & 1.53 & implicated in regulation of intestinal mucosal defence \\
\hline 101753_s_at & Lyzs, Lzp-s & $2 \cdot 20^{*}$ & 1.60 & $\begin{array}{l}\text { catalyses the hydrolysis of certain mucopolysaccharides } \\
\text { of bacterial cell walls }\end{array}$ \\
\hline 100611_at & Lyzs & $1.91^{*}$ & 1.48 & \\
\hline 92735_at & Pla2g2a & $2 \cdot 02^{*}$ & 1.58 & $\begin{array}{l}\text { phospholipase, may contribute to the gastric response to } \\
\text { bacterial infection }\end{array}$ \\
\hline 101752_f_at & $\operatorname{lgh}-6$ & 1.05 & $1.74^{*}$ & $\lg$ heavy chain of IgM \\
\hline 160909_at & Sprr1a & $1 \cdot 15$ & $1 \cdot 45^{\star}$ & $\begin{array}{l}\text { implicated in fortifying the intestinal epithelial barrier in } \\
\text { response to bacterial colonization }\end{array}$ \\
\hline \multicolumn{5}{|l|}{ Miscellaneous } \\
\hline $93142 \_$at & Bach1 & 1.75 & $1 \cdot 50^{*}$ & $\begin{array}{l}\text { forms heterodimers with MafK and coordinates transcription } \\
\text { activation and repression by this factor }\end{array}$ \\
\hline 92811_at & Rbp2 & 1.47 & $1.57^{*}$ & regulates the uptake and metabolism of vitamin $\mathrm{A}$ \\
\hline 104155_f_at & Atf3 & $1.46^{*}$ & $1 \cdot 55^{*}$ & $\begin{array}{l}\text { a member of the mammalian activation transcription } \\
\text { factor/CREB protein family of transcription factors }\end{array}$ \\
\hline 94910_at & Nde1 & $2 \cdot 13^{* *}$ & -1.06 & function in the intestine poorly understood \\
\hline 160906 i at & - & $1.71^{*}$ & 1.28 & - \\
\hline 162190_r_at & Lmbr1I & $1.65^{*}$ & 1.40 & function poorly understood \\
\hline 96679_at & Dnajb9 & $1.52^{*}$ & 1.27 & function poorly understood \\
\hline 160829_at & Phlda1 & $1.37^{\star}$ & 1.49 & function poorly understood \\
\hline $92470 \_$f_at & LOC546230 & 1.20 & $1.49^{*}$ & - \\
\hline 101704_at & $H n f 4 g$ & 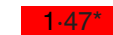 & 1.40 & transcription factor involved in divergent functions \\
\hline 93975_at & Errfi1 & $1 \cdot 15$ & $1.45^{*}$ & function poorly understood \\
\hline 102208_at & St3gal6 & $-1.91^{*}$ & -1.35 & $\begin{array}{l}\text { catalyses the transfer of sialic acid to terminal positions on the } \\
\text { carbohydrate groups of glycoproteins and glycolipids }\end{array}$ \\
\hline 95586_at & P2rx4 & $-1.51^{*}$ & $-1.41^{*}$ & ligand-gated ion channel \\
\hline 95518_at & 1810015C04Rik & $-1.50^{*}$ & $-1 \cdot 31$ & - \\
\hline 93372 at & Anp32a & $-1.47^{\star}$ & $-1 \cdot 29^{\star}$ & a putative HLA class II-associated protein \\
\hline 96656_at & Wdr48 & $-1.43^{*}$ & -1.33 & function poorly understood \\
\hline 96088 at & Ndrg2 & $-1.42^{*}$ & -1.25 & function in the intestine poorly understood \\
\hline
\end{tabular}

Values are significantly different, determined by two-sided Student's $t$ test $\left({ }^{\star} P \leq 0.05 ;{ }^{* \star} P \leq 0.01 ;{ }^{* \star *} P \leq 0.005\right)$.

† Global mRNA expression pattern was characterized in distal ileum using Affymetrix (Santa Clara, CA, USA) gene arrays. The filtering criteria were set to a mean absolute fold change $>1.41$ ( $\log _{2}$ fold change $>0.5$ ) and a $P$ value $<0.05$ in either of the two test groups, compared with the control group. In addition, the mean intensity in the group showing highest expression should be $>75$.

$\ddagger$ For details of procedures, see Materials and methods.

The colours represent the differential expression pattern. Green indicates down-regulation; red indicates up-regulation with an absolute log ${ }_{2}$ fold change $>0.5$. CREB, cyclic AMP-response element-binding; HLA, histocompatibility locus antigen.

Lactobacillus bacteria have been shown to improve clinical symptoms of inflammatory bowel disease, a major clinical problem in the field of gastroenterology (Matsumoto et al. 2001; Saggioro, 2004). In this context, it is interesting to note that Serpinal, encoding $\alpha 1$-antitrypsin, was up-regulated in the NMF mice in response to Lactobacillus administration (Table 2). The concentration of $\alpha 1$-antitrypsin is increased in the intestine in connection with inflammatory bowel disease, where its release is thought to protect the mucosa from proteolytic damage (Faust et al. 2002). We hypothesize that increase in Serpinal transcription may be part of the mechanism for Lactobacillus bacteria to reduce the severity of inflammatory bowel disease, which remains to be addressed in further studies.

Recently, the role of factors secreted from the gut in regulation of food intake and whole body energy partitioning has been acknowledged (Bloom et al. 2005). Also, connection between gut microflora and energy homeostasis of the host organism has become recognized. Colonization of GF mice with normal gut microflora was shown to increase body fat and cause insulin resistance (Backhed et al. 2004). Conversely, obesity affects the composition of the gut microbiota in mice (Ley et al. 2005). Interestingly, consumption of dairy products supplemented with Lactobacillus bacteria has been shown to decrease serum cholesterol and LDL-cholesterol (Akalin et al. 1997). However, the mechanisms responsible for directing these changes remain largely unknown. In this context, one of the most interesting findings of the present study was the coordinated differential regulation of transcripts for several secreted factors controlling whole body lipid and glucose metabolism, in response to the administration of Lactobacillus in GF mice. Adiponectin (Adipoq) and adipsin 

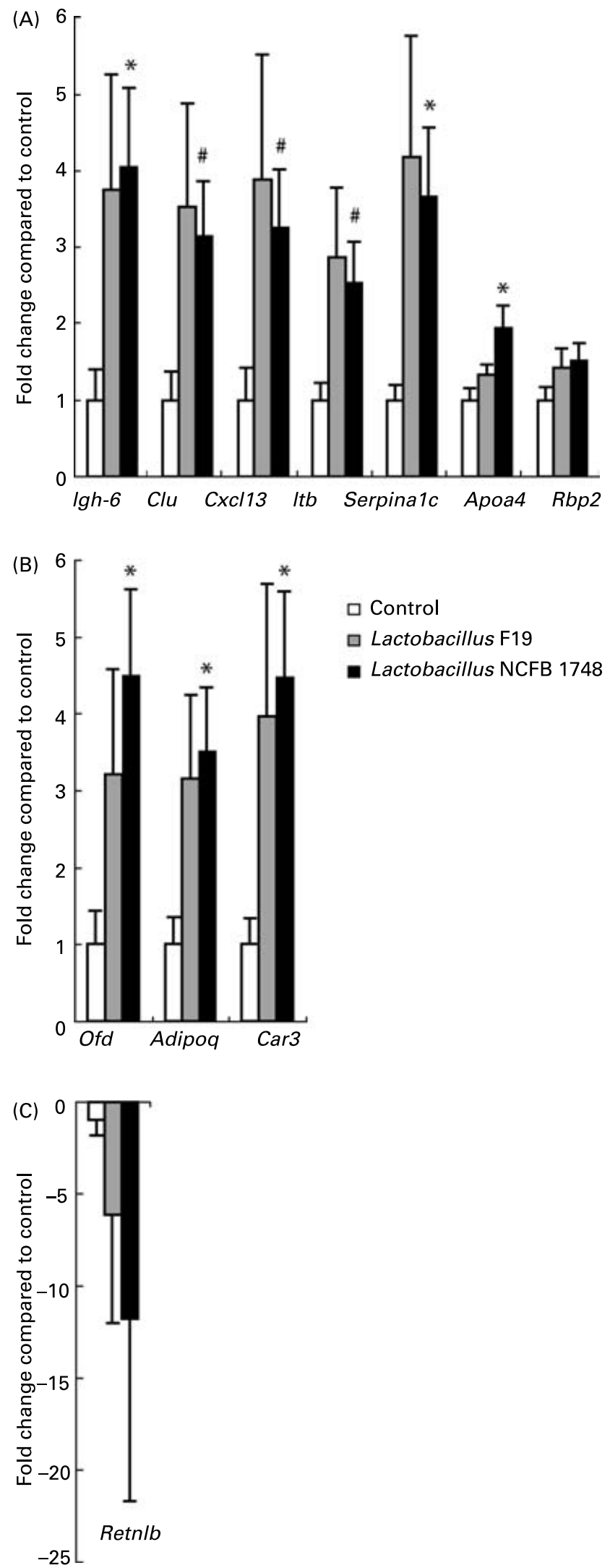

Fig. 1. Expression analysis by quantitative real-time $P C R$ in (A) normal microflora and (B), (C) germ-free mice receiving Lactobacillus paracasei ssp. F19 (L. F19; 司) or Lactobacillus acidophilus NCFB 1748 (L. NCFB 1748; $\mathbf{\square})$ compared with the control group of mice $(\square)$ receiving placebo product.
$(C f d)$, both up-regulated in the test groups of mice, are known to be decreased in overweight human subjects and/or in animal models of obesity (Flier et al. 1987; Lowell et al. 1990; Shillabeer et al. 1992; Hu et al. 1996; Yamauchi et al. 2001). Interestingly, pharmacological adiponectin treatment in rodents has been shown to increase insulin sensitivity (Pajvani $\&$ Scherer, 2003). Resistin like $\beta$ (Retnlb), on the other hand, was down-regulated in the test groups of mice. Recently, elevated serum levels of resistin like $\beta$, attributable to increased mRNA and protein production in intestine and bone marrow, were reported in mice fed a high-fat diet as well as in animal models of type 2 diabetes (Shojima et al. 2005). Infusion of resistin like $\beta$ has been shown to induce insulin resistance (Rajala et al. 2003). In the light of this knowledge, an increased signal for adiponectin and adipsin in combination with reduced expression of resistin like $\beta$, observed in GF mice in response to the delivery of Lactobacillus (Table 3), suggests the possibility for improved insulin sensitivity of the host organism. The present report does not answer the question if similar host responses are elicited by other components of the gut microflora. Notably, colonization of GF mice with normal gut microflora has been shown to decrease, rather than increase, insulin sensitivity (Backhed et al. 2004). One factor suppressed in the ileum in response to conventionalization of gnotobiotic mice, implicated in the promotion of adiposity and insulin resistance, is Fiaf (Backhed et al. 2004). Probe sets for Fiaf were represented on the Affymetrix microarray used in this study. However, the mRNA level for this gene was unaltered in response to Lactobacillus administration (data not shown). Interestingly, mono-colonization of GF mice with common gut bacterium Bacteroides thetaiotaomicron (Hooper et al. 2001) did not evoke effects similar to Lactobacillus on the expression of genes involved in regulation of energy homeostasis. This suggests that the gut microbiota influence the expression of genes important for energy metabolism differently depending on the microbial composition and that Lactobacilli may influence transcription of genes involved in regulation of energy homeostasis in a favourable way. However, notice should be taken that different strains of GF mice have been used in the reports compared (C57BL/6J, NMRI/KI or Swiss Webster strain was used by Backhed et al. 2004, Hooper et al. 2001 or in the present study, respectively) and, therefore, we cannot exclude the possibility that the differences in the transcriptional changes described in response to the different bacterial strains are at least partly related to the variation in the genetic background of the host.

Adiponectin, adipsin and resistin like $\beta$ were differentially regulated in response to Lactobacillus administration in GF but not in NMF mice. The gut microbiota are known to

\footnotetext{
The relative quantities of different mRNA transcripts were calculated after normalization of the data against an endogenous control - acidic ribosomal phosphoprotein PO (Arbp). The results are shown as the fold change of expression in two test groups relative to the corresponding control group, with the expression level in the control groups set to 1 (A),(B) or -1 (C). Quantitative real-time PCR analysis was performed on the same set of samples that was used in the gene array experiment. Statistical differences were determined by two-sided Student's $t$ test: ${ }^{\star} P \leq 0.05$; $\# P<0.06$. For details of procedures, see Materials and methods.
} 
(A)

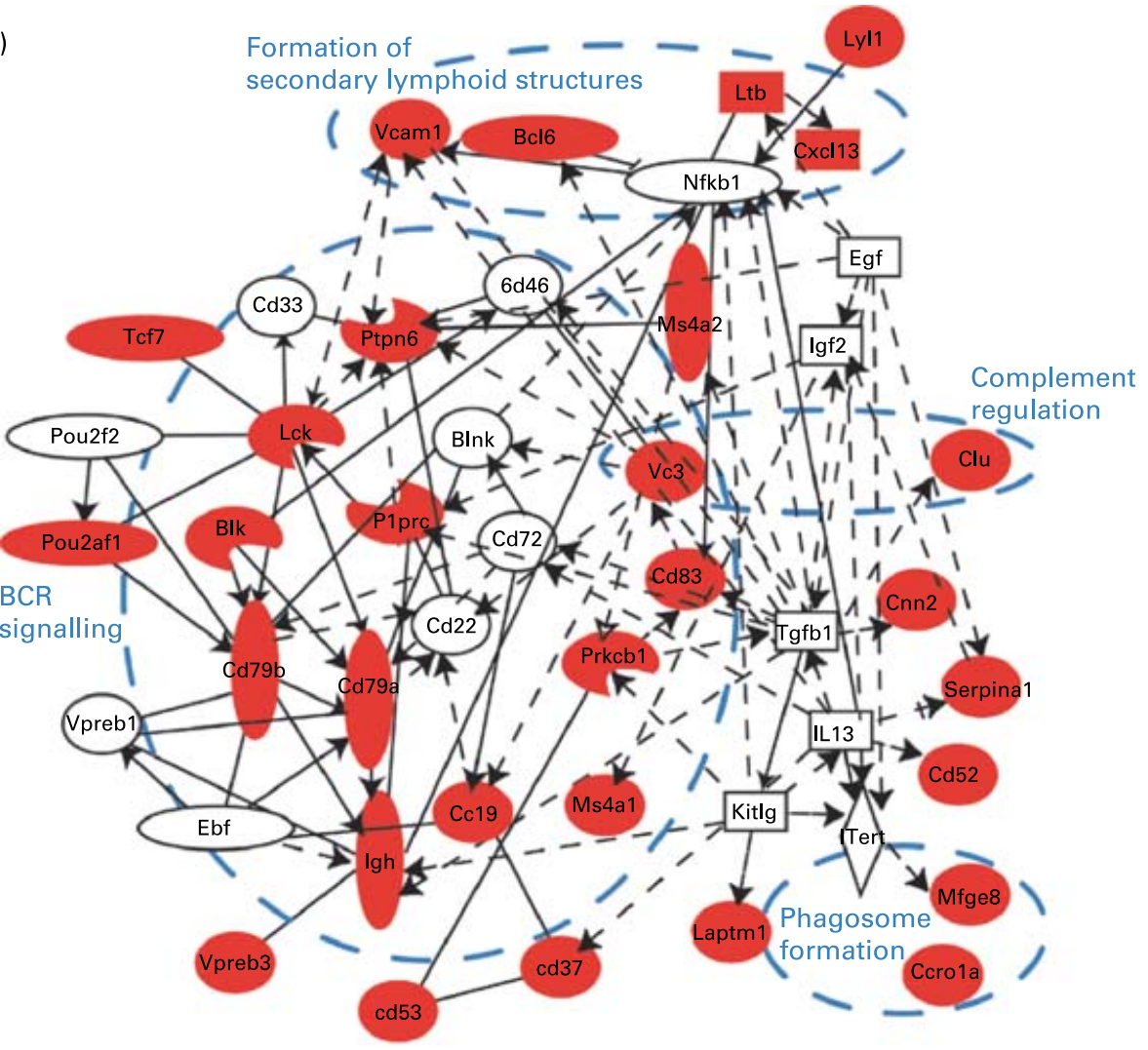

(B)

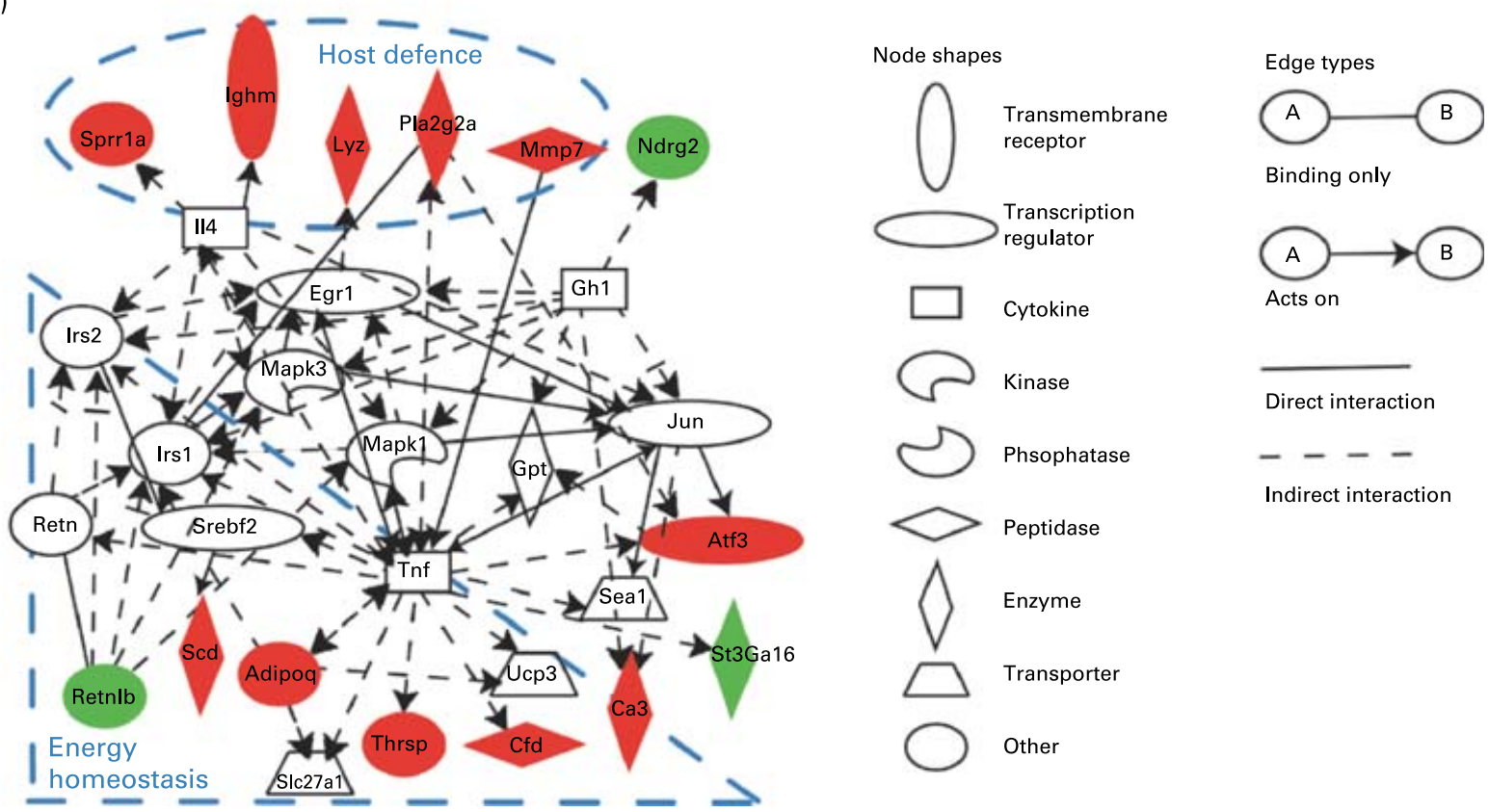

Fig. 2. The Affymetrix Probe Set IDs (Santa Clara, CA, USA) for genes listed in Tables 2 and 3 were imported into the Ingenuity Pathway Analysis software (Ingenuity Systems, Redwood City, CA, USA), to generate interactome and transcriptional networks connecting the query genes and all other gene products. The networks generated by the Ingenuity Pathway Analysis tool are ranked by score reflecting on how relevant they are to the genes within the dataset. For normal microflora dataset, the two networks with the highest score contained a number of common focus genes and were merged into a single network (A). For germfree dataset, the network with the highest score containing fourteen focus genes is presented (B). Red and green nodes are input genes with red indicating upregulation and green indicating down-regulation in mice receiving Lactobacillus compared with the control group of mice receiving placebo product (Tables 2 and 3). The white nodes indicate genes not part of the dataset file. The common biological function for selected groups of nodes is shown. Some peripheral nodes and connection genes were removed for simplicity. The displayed network features only genes with functional interactions. BCR, B cell receptor. For details of procedures, see Materials and methods. 
regulate the intestinal motility, differentiation, assembly of gut-associated lymphoid tissue, etc., resulting in substantial differences comparing the morphology and function of gnotobiotic v. NMF intestine (Falk et al. 1998). Additionally, Lactobacillus bacteria have been shown to colonize the intestine of both GF and NMF mice (Bateup et al. 1995; Filho-Lima et al. 2000). However, the colonization efficiency might differ comparing the gnotobiotic $v$. NMF gut. Therefore, it is not surprising that the changes in gene expression pattern caused by Lactobacillus administration in GF mice differ from the responses in NMF mice. Further studies are required to clarify if the differential expression of Adipoq, Cfd and Retnlb observed in GF mice can be evoked in NMF conditions applying different product administration regimes and/or dose of probiotics. Also, the influence of genetic background and/or species differences of the host on the effect of Lactobacillus administration remains to be addressed.

Gene array approaches are limited by multiple comparison caveats and, therefore, a certain number of false-positive results can be expected. Also, several of the alterations in gene expression that we describe are of relatively low magnitude and of marginal significance. However, the biological relevance of the differentially regulated targets highlighted in the present report is supported by the fact that a number of genes from the same biological pathway are coordinately changed as illustrated by Ingenuity Pathway Analysis (Fig. 2). Furthermore, the two genetically close Lactobacillus strains used in the study evoke similar changes in the expression profile of the genes belonging to these functional groups, which further emphasizes the biological interpretation of the data. Finally, the differential expression of selected targets was confirmed by an alternative approach, quantitative real-time PCR, which largely supported the conclusions from the gene array experiments (Fig. 1).

In summary, the present study characterizes global transcriptional responses to administration of two probiotic strains

- L. F19 and L. acidophilus NCFB 1748 - in vivo. The results reveal that Lactobacillus bacteria modulate expression of host genes participating in different fundamental physiological processes in the intestine. Several changes in gene expression that we describe were expected, being consistent with the earlier publications in the field, e.g. stimulation of the immune system in NMF mice and increased expression of host defence markers in GF mice. Additionally, we report differential expression of several genes previously not known to be regulated by Lactobacillus, such as transcripts involved in regulation of energy homeostasis. The differential response in NMF $v$. GF mice that we describe underlines the fact that host-bacterial interactions are both complex and dynamic, and any impact of Lactobacillus feeding is likely to be affected by factors such as age, sex, health status, already existing gut microflora, etc. Consequently, additional molecular and physiological studies are required to characterize the functional impact of the changes in transcriptional profile presented in the present report.

\section{Acknowledgements}

The study was supported by Arla Foods, Innovation and Arexis Research and Development Fund. The authors are grateful to
Professor Lora V. Hooper and Professor Jeffrey I. Gordon for kindly providing an access to the raw data files from their previously published study (Hooper et al. 2001). Dr Robert Brooksbank (Cambridge Biotechnology Ltd.) is acknowledged for generating the Ingenuity Pathway Analysis maps.

\section{References}

Akalin AS, Gonc S \& Duzel S (1997) Influence of yogurt and acidophilus yogurt on serum cholesterol levels in mice. J Dairy Sci 80, 2721-2725.

Applied Biosystems (1997) User Bulletin \#2 ABI PRISM 7700 Sequence Detection System. Foster City, CA: Applied Biosystems.

Backhed F, Ding H, Wang T, Hooper LV, Koh GY, Nagy A, Semenkovich CF \& Gordon JI (2004) The gut microbiota as an environmental factor that regulates fat storage. Proc Natl Acad Sci USA 101, 15718-15723.

Bateup JM, McConnell MA, Jenkinson HF \& Tannock GW (1995) Comparison of Lactobacillus strains with respect to bile salt hydrolase activity, colonization of the gastrointestinal tract, and growth rate of the murine host. Appl Environ Microbiol 61, 1147-1149.

Bloom S, Wynne K \& Chaudhri O (2005) Gut feeling - the secret of satiety? Clin Med 5, 147-152.

Di Caro S, Tao H, Grillo A, Elia C, Gasbarrini G, Sepulveda AR \& Gasbarrini A (2005) Effects of Lactobacillus GG on genes expression pattern in small bowel mucosa. Dig Liver Dis 37, 320-329.

Falk PG, Hooper LV, Midtvedt T \& Gordon JI (1998) Creating and maintaining the gastrointestinal ecosystem: what we know and need to know from gnotobiology. Microbiol Mol Biol Rev 62, $1157-1170$.

Faust D, Raschke K, Hormann S, Milovic V \& Stein J (2002) Regulation of alpha1-proteinase inhibitor release by proinflammatory cytokines in human intestinal epithelial cells. Clin Exp Immunol 128, 279-284.

Filho-Lima JV, Vieira EC \& Nicoli JR (2000) Antagonistic effect of Lactobacillus acidophilus, Saccharomyces boulardii and Escherichia coli combinations against experimental infections with Shigella flexneri and Salmonella enteritidis subsp. typhimurium in gnotobiotic mice. J Appl Microbiol 88, 365-370.

Flier JS, Cook KS, Usher P \& Spiegelman BM (1987) Severely impaired adipsin expression in genetic and acquired obesity. Science 237, 405-408.

Galdeano CM \& Perdigon G (2006) The probiotic bacterium Lactobacillus casei induces activation of the gut mucosal immune system through innate immunity. Clin Vaccine Immunol 13, 219-226.

Hooper LV, Wong MH, Thelin A, Hansson L, Falk PG \& Gordon JI (2001) Molecular analysis of commensal host-microbial relationships in the intestine. Science 291, 881-884.

Hu E, Liang P \& Spiegelman BM (1996) AdipoQ is a novel adiposespecific gene dysregulated in obesity. J Biol Chem 271, $10697-10703$.

Ibnou-Zekri N, Blum S, Schiffrin EJ \& von der Weid T (2003) Divergent patterns of colonization and immune response elicited from two intestinal Lactobacillus strains that display similar properties in vitro. Infect Immun 71, 428-436.

Kinlaw WB, Church JL, Harmon J \& Mariash CN (1995) Direct evidence for a role of the 'spot 14' protein in the regulation of lipid synthesis. J Biol Chem 270, 16615-16618.

Levin MS (1993) Cellular retinol-binding proteins are determinants of retinol uptake and metabolism in stably transfected Caco-2 cells. J Biol Chem 268, 8267-8276.

Ley RE, Backhed F, Turnbaugh P, Lozupone CA, Knight RD \& Gordon JI (2005) Obesity alters gut microbial ecology. Proc Natl Acad Sci USA 102, 11070-11075. 
Lissoos TW, Davis AE \& Levin MS (1995) Vitamin A trafficking in Caco-2 cells stably transfected with cellular retinol binding proteins. Am J Physiol 268, G224-G231.

Lowell BB, Napolitano A, Usher P, Dulloo AG, Rosen BS, Spiegelman BM \& Flier JS (1990) Reduced adipsin expression in murine obesity: effect of age and treatment with the sympathomimeticthermogenic drug mixture ephedrine and caffeine. Endocrinology 126, $1514-1520$.

Matsumoto S, Watanabe N, Imaoka A \& Okabe Y (2001) Preventive effects of Bifidobacterium- and Lactobacillus-fermented milk on the development of inflammatory bowel disease in senescenceaccelerated mouse P1/Yit strain mice. Digestion 64, 92-99.

Mättö J, Fondén R, Tolvanen T, von Wright A, Vilpponen-Salmela T, Satokari R \& Saarela M (2006) Intestinal survival and persistence of probiotic Lactobacillus and Bifidobacterium strains administered in triple-strain yoghurt. Int Dairy J 16, 1174-1180.

Pajvani UB \& Scherer PE (2003) Adiponectin: systemic contributor to insulin sensitivity. Curr Diab Rep 3, 207-213.

Perdigon G, Vintini E, Alvarez S, Medina M \& Medici M (1999) Study of the possible mechanisms involved in the mucosal immune system activation by lactic acid bacteria. J Dairy Sci $\mathbf{8 2}$, $1108-1114$.

Rajala MW, Obici S, Scherer PE \& Rossetti L (2003) Adiposederived resistin and gut-derived resistin-like molecule-beta selectively impair insulin action on glucose production. $J$ Clin Invest 111, 225-230.

Roberfroid MB (2000) Prebiotics and probiotics: are they functional foods? Am J Clin Nutr 71, 1682S-1687S, discussion 1688S$1690 \mathrm{~S}$.
Saggioro A (2004) Probiotics in the treatment of irritable bowel syndrome. J Clin Gastroenterol 38, S104-S106.

Saris WH, Asp NG, Bjorck I, et al. (1998) Functional food science and substrate metabolism. Br J Nutr 80, Suppl. 1, S47-S75.

Shillabeer G, Hornford J, Forden JM, Wong NC, Russell JC \& Lau DC (1992) Fatty acid synthase and adipsin mRNA levels in obese and lean JCR:LA-cp rats: effect of diet. J Lipid Res 33, 31-39.

Shojima N, Ogihara T, Inukai K, et al. (2005) Serum concentrations of resistin-like molecules beta and gamma are elevated in high-fat-fed and obese $\mathrm{db} / \mathrm{db}$ mice, with increased production in the intestinal tract and bone marrow. Diabetologia 48, 984-992.

Shu Q \& Gill HS (2002) Immune protection mediated by the probiotic Lactobacillus rhamnosus HN001 (DR20) against Escherichia coli O157:H7 infection in mice. FEMS Immunol Med Microbiol 34, 59-64.

Stanton LW, Ponte PA, Coleman RT \& Snyder MA (1991) Expression of CA III in rodent models of obesity. Mol Endocrinol 5, 860-866.

Sullivan A, Barkholt L \& Nord CE (2003) Lactobacillus acidophilus, Bifidobacterium lactis and Lactobacillus F19 prevent antibioticassociated ecological disturbances of Bacteroides fragilis in the intestine. J Antimicrob Chemother 52, 308-311.

Wagner RD, Warner T, Roberts L, Farmer J \& Balish E (1997) Colonization of congenitally immunodeficient mice with probiotic bacteria. Infect Immun 65, 3345-3351.

Yamauchi T, Kamon J, Waki H, et al. (2001) The fat-derived hormone adiponectin reverses insulin resistance associated with both lipoatrophy and obesity. Nat Med 7, 941-946. 